\title{
Voltage Profile Enhancement and Reduction of Real Power Loss by Hybrid Biogeography Based Artificial Bee Colony algorithm
}

\author{
K Lenin, B Ravindhranath Reddy \\ Jawaharlal Nehru Technological University Kukatpally, \\ Hyderabad 500 085, India. \\ e-mail: ${ }^{1}$ gklenin@gmail.com
}

\begin{abstract}
This paper presents Hybrid Biogeography algorithm for solving the multi-objective reactive power dispatch problem in a power system. Real Power Loss minimization and maximization of voltage stability margin are taken as the objectives. Artificial bee colony optimization $(A B C)$ is quick and forceful algorithm for global optimization. Biogeography-Based Optimization (BBO) is a new-fangled biogeography inspired algorithm. It mainly utilizes the biogeography-based relocation operator to share the information among solutions. In this work, a hybrid algorithm with $B B O$ and $A B C$ is projected, and named as HBBABC (Hybrid Biogeography based Artificial Bee Colony Optimization), for the universal numerical optimization problem. $H B B A B C$ merge the searching behavior of $A B C$ with that of $B B O$. Both the algorithms have different solution probing tendency like $A B C$ have good exploration probing tendency while $B B O$ have good exploitation probing tendency. $\mathrm{HBBABC}$ used to solve the reactive power dispatch problem and the proposed technique has been tested in standard IEEE30 bus test system.
\end{abstract}

Keywords: Modal analysis, optimal reactive power, Transmission loss, Artificial Bee Colony Algorithm, Hybrid Biogeography

\section{Introduction}

Optimal reactive power dispatch problem is one of the hard optimization problems in power system. The problem that has to be solved in a reactive power optimization is to decide on the required reactive generation at various locations so as to optimize the objective function. Here the reactive power dispatch problem engages about best utilization of the existing generator bus voltage magnitudes, transformer tap setting and the output of reactivepower sources so as to minimize the real power loss and to augment the voltage stability of the system. Various mathematical techniques have been implemented to solve this optimal reactive power dispatch problem. These include the gradient method [1-2], Newton method [3] and linear programming [4-7].The gradient andNewton methods experience from the intricacy in handling inequality constraints. Recently global Optimization techniques such as genetic algorithmshave been proposed to solve the reactive power flow problem [8, 9]. Hybridization of algorithm means to merge the capabilities of different algorithm in a single algorithm. Hybridization is done to triumph over the drawback in the existing algorithms and to obtain superior solutions. Evolutionary Algorithms (EAs) are muchadmired for the hybridization due to their dissimilar capabilities in handling different types of problems. Continuous research is going on to find new optimization techniques which are able to handling variety of problems with high effectiveness, efficiency and flexibility and thus there are many such optimization algorithms like GA, SA, DE, PSO, ACO, SFLA, ABC, BBO etc. Hybridization is one of the admired methods to augment the effectiveness, efficiency and flexibility of the algorithm to create better solution and convergence rates and minimizing computational times. Many such amalgam algorithms are available in the literature and continuous efforts are sustained to develop new hybrid algorithms. ABC (artificial bee colony) [15]-[18] is a straightforward and commanding population-based algorithm for finding the global optimum solutions. $A B C$ separate the population in two key parts viz. employed bees and onlooker bees. Employed bees begin explore with precise rules and onlooker bees go behind the employed bees in analogous to the fitness of employed bees and it also revise the solution as employed bees. If there is no change in the fitness of employed bees for some number of generations then that bee is transformed in scout bee which starts for a 
new-fangled search and acts as an employed bee from then. Algorithm prolong for predefined number of generations or until the best solution is found. So ABC finds the universal solution by exploring the search space with specific rules followed by employed bees, onlooker bees and scout bees. Biogeography-Based Optimization (BBO), proposed by Simon [27], anovel global optimization algorithm based on the biogeography theory, and it is the study about the distribution of species. BBO [28]-[30] is also population-based optimization system. In the original BBO algorithm, every solution of the population is a vector of integers. BBO updates the solution subsequent to immigration and emigration phenomenon of the species from one place to the other which is referred as islands by Simon. BBO has high-quality exploitation ability as solution and is updated by exchanging the existing design variables among the solution. In order to merge the searching capabilities of $A B C$ and $B B O$, in this paper, we plan a hybrid $A B C$ with $\mathrm{BBO}$, referred to as $\mathrm{HBBABC}$, for solving the optimal reactive power dispatch problem. In HBBABC, algorithm begins by updating the solutions utilizing the immigration and emigration rates. Solution is further customized by using the exploration propensity of $A B C$ using employed, onlooker and scout bees.

\section{Voltage Stability Evaluation}

\section{Modal analysis for voltage stability evaluation}

The linearized steady state system power flow equations are given by,

$$
\left[\begin{array}{l}
\Delta \mathrm{P} \\
\Delta \mathrm{Q}
\end{array}\right]=\left[\begin{array}{c}
\mathrm{J}_{\mathrm{p} \theta} \mathrm{J}_{\mathrm{pv}} \\
\mathrm{J}_{\mathrm{q} \theta} \mathrm{J}_{\mathrm{QV}}
\end{array}\right]
$$

Where

$\Delta \mathrm{P}=$ Incremental change in bus real power.

$\Delta Q=$ Incremental change in bus reactive

Power injection

$\Delta \theta=$ incremental change in bus voltage angle.

$\Delta \mathrm{V}=$ Incremental change in bus voltage Magnitude

$\mathrm{Jp} \theta, \mathrm{J} P V, J \mathrm{Q} \theta, \mathrm{J} Q \mathrm{~V}$ jacobian matrix are the sub-matrixes of the System voltage stability is affected by both $\mathrm{P}$ and $\mathrm{Q}$. On the other hand at each operational point we keep $\mathrm{P}$ constant and evaluate voltage stability by taking into accountof incremental relationship between $Q$ and $V$.

To reduce (1), let $\Delta P=0$, then.

$$
\begin{aligned}
& \Delta \mathrm{Q}=\left[\mathrm{J}_{\mathrm{QV}}-\mathrm{J}_{\mathrm{Q} \theta} \mathrm{J}_{\mathrm{P} \theta^{-1}} \mathrm{~J}_{\mathrm{PV}}\right] \Delta \mathrm{V}=\mathrm{J}_{\mathrm{R}} \Delta \mathrm{V} \\
& \Delta \mathrm{V}=\mathrm{J}^{-1}-\Delta \mathrm{Q}
\end{aligned}
$$

Where

$\mathrm{J}_{\mathrm{R}}=\left(\mathrm{J}_{\mathrm{QV}}-\mathrm{J}_{\mathrm{Q} \theta} \mathrm{J}_{\mathrm{P} \theta^{-1}} \mathrm{JPV}\right)$

$\mathrm{J}_{\mathrm{R}}$ is called the reduced Jacobian matrix of the system.

Modes of Voltage instability:

Voltage Stability characteristics are computed by the Eigen values and Eigen vectors.

Let

$$
\mathrm{J}_{\mathrm{R}}=\xi \wedge \eta
$$

Where,

$\xi=$ right eigenvector matrix of $\mathrm{JR}$

$\eta=$ left eigenvector matrix of $\mathrm{JR}$

$\Lambda=$ diagonal Eigen value matrix of $\mathrm{JR}$ and 


$$
\mathrm{J}_{\mathrm{R}^{-1}}=\xi \wedge^{-1} \eta
$$

From (3) and (6), we have

$$
\Delta \mathrm{V}=\xi \wedge^{-1} \eta \Delta \mathrm{Q}
$$

or

$$
\Delta \mathrm{V}=\sum_{\mathrm{I}} \frac{\xi_{\mathrm{i}} \eta_{\mathrm{i}}}{\lambda_{\mathrm{i}}} \Delta \mathrm{Q}
$$

Where $\xi i$ is the ith column right Eigen vector and $\eta$ the ith row left eigenvector of JR.

$\lambda i$ is the ith eigen value of JR.

The ith modal reactive power variation is,

$$
\Delta \mathrm{Q}_{\mathrm{mi}}=\mathrm{K}_{\mathrm{i}} \xi_{\mathrm{i}}
$$

where,

$$
\mathrm{K}_{\mathrm{i}}=\sum_{\mathrm{j}} \xi_{\mathrm{ij}}{ }^{2}-1
$$

Where

$\xi j i$ is the jth element of $\xi i$

The corresponding ith modal voltage variation is

$$
\Delta \mathrm{V}_{\mathrm{mi}}=\left[1 / \lambda_{\mathrm{i}}\right] \Delta \mathrm{Q}_{\mathrm{mi}}
$$

In (8), let $\Delta Q=$ ek where ek has all its elements zero except the kth one being 1. Then,

$$
\Delta \mathrm{V}=\sum_{\mathrm{i}} \frac{\eta_{1 \mathrm{k} \xi_{1}}}{\lambda_{1}}
$$

$\eta_{1 \mathrm{k}} \mathrm{k}$ th element of $\eta_{1}$

$V-Q$ sensitivity at bus $k$

$$
\frac{\partial \mathrm{V}_{\mathrm{K}}}{\partial \mathrm{Q}_{\mathrm{K}}}=\sum_{\mathrm{i}} \frac{\eta_{1 \mathrm{k}} \xi_{1}}{\lambda_{1}}=\sum_{\mathrm{i}} \frac{\mathrm{P}_{\mathrm{ki}}}{\lambda_{1}}
$$

\section{Problem Formulation}

The objective of the reactive power dispatch problem considered here is to reduce the real power loss and to enhance the static voltage stability margins (SVSM).

\section{Minimization of Real Power Loss}

Minimization of the real power loss (Ploss) in transmission lines of a power system is mathematically stated as follows.

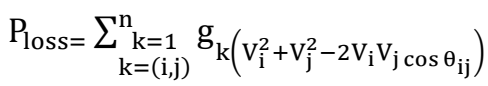

Where $\mathrm{n}$ is the number of transmission lines, $\mathrm{gk}$ is the conductance of branch $\mathrm{k}, \mathrm{Vi}$ and $\mathrm{Vj}$ are voltage magnitude at bus $\mathrm{i}$ and bus $\mathrm{j}$, and $\theta \mathrm{ij}$ is the voltage angle difference between bus $\mathrm{i}$ and bus j.

\section{Minimization of Voltage Deviation}

Minimization of the Deviations in voltage magnitudes (VD) at load buses is mathematically stated as follows. 
Minimize VD $=\sum_{\mathrm{k}=1}^{\mathrm{nl}}\left|\mathrm{V}_{\mathrm{k}}-1.0\right|$

Where $\mathrm{nl}$ is the number of load busses and $\mathrm{Vk}$ is the voltage magnitude at bus $\mathrm{k}$.

\section{System Constraints}

Objective functions are subjected to these constraints shown below.

Load flow equality constraints:

$$
\begin{aligned}
& P_{G i}-P_{D i}-V_{i} \sum_{j=1}^{n b} V_{j}\left[\begin{array}{cc}
G_{i j} & \cos \theta_{i j} \\
+B_{i j} & \sin \theta_{i j}
\end{array}\right]=0, i=1,2 \ldots, n b \\
& Q_{G i}-Q_{D i} V_{i} \sum_{j=1}^{n b} V_{j}\left[\begin{array}{cc}
G_{i j} & \cos \theta_{i j} \\
+B_{i j} & \sin \theta_{i j}
\end{array}\right]=0, i=1,2 \ldots, n b
\end{aligned}
$$

where, $\mathrm{nb}$ is the number of buses, $\mathrm{PG}$ and $\mathrm{QG}$ are the real and reactive power of the generator, $\mathrm{PD}$ and $\mathrm{QD}$ are the real and reactive load of the generator, and $\mathrm{Gij}$ and $\mathrm{Bij}$ are the mutual conductance and susceptance between bus $\mathrm{i}$ and bus $\mathrm{j}$.

Generator bus voltage (VGi) inequality constraint:

$$
\mathrm{V}_{\mathrm{Gi}}^{\min } \leq \mathrm{V}_{\mathrm{Gi}} \leq \mathrm{V}_{\mathrm{Gi}}^{\max }, \mathrm{i} \in \mathrm{ng}
$$

Load bus voltage (VLi) inequality constraint:

$$
\mathrm{V}_{\mathrm{Li}}^{\min } \leq \mathrm{V}_{\mathrm{Li}} \leq \mathrm{V}_{\mathrm{Li}}^{\max }, \mathrm{i} \in \mathrm{nl}
$$

Switchable reactive power compensations (QCi) inequality constraint:

$$
\mathrm{Q}_{\mathrm{Ci}}^{\min } \leq \mathrm{Q}_{\mathrm{Ci}} \leq \mathrm{Q}_{\mathrm{Ci}}^{\max }, \mathrm{i} \in \mathrm{nc}
$$

Reactive power generation (QGi) inequality constraint:

$$
\mathrm{Q}_{\mathrm{Gi}}^{\min } \leq \mathrm{Q}_{\mathrm{Gi}} \leq \mathrm{Q}_{\mathrm{Gi}}^{\max }, \mathrm{i} \in \mathrm{ng}
$$

Transformers tap setting (Ti) inequality constraint:

$$
\mathrm{T}_{\mathrm{i}}^{\mathrm{min}} \leq \mathrm{T}_{\mathrm{i}} \leq \mathrm{T}_{\mathrm{i}}^{\max }, \mathrm{i} \in \mathrm{nt}
$$

Transmission line flow (SLi) inequality constraint:

$$
\mathrm{S}_{\mathrm{Li}}^{\min } \leq \mathrm{S}_{\mathrm{Li}}^{\max }, \mathrm{i} \in \mathrm{nl}
$$

Where, nc, ng and nt are numbers of the switchable reactive power sources, generators and transformers.

\section{Biogeography-Based Optimization (BBO)}

BBO is a new population-based optimization algorithm stimulated by the natural biogeography distribution of dissimilar species. In BBO, everyentity is considered as a "habitat" with a habitat suitability index (HIS). A high-quality solution is analogous to an island with a high $\mathrm{HSI}$, and a poor solution indicates an island with a low HSI. High HSI solutions are inclined to share their features with low HSI solutions. Low HSI solutions admit lot of novel features from high $\mathrm{HSI}$ solutions. In BBO, each individual has its own immigration rate $\lambda$ and emigration rate $\mu$. A high-quality solution has higher $\mu$ and lower $\lambda$ and vice versa. The immigrant ion rate and the emigration rate are functions of the amount of species in the habitat. They can be calculated as follows, 


$$
\begin{aligned}
& \lambda_{\mathrm{k}}=\mathrm{I}\left(1-\frac{\mathrm{k}}{\mathrm{n}}\right) \\
& \mu_{\mathrm{k}}=\mathrm{E}\left(\frac{\mathrm{k}}{\mathrm{n}}\right)
\end{aligned}
$$

Where $\mathrm{I}$ is the maximum possible immigration rate; $\mathrm{E}$ is the maximum possible emigration rate; $\mathrm{k}$ is the number of species of the $\mathrm{k}$-th individual; and $\mathrm{n}$ is the maximum number of species. In $\mathrm{BBO}$, there are two key operators, the migration and the mutation.

\section{Migration}

Consider a population of contestant which is represented by a design variable. Every design variable for particular population member is measured as SIV for that population member. Every population member is considered as individual habitat or Island. The objective function value point out the $\mathrm{HSI}$ for the particular population member. The emigration and immigration rates of every solution are used to probabilistically distribute information between habitats. If a given solution is chosen for modification, then its immigration rate $\lambda$ is used to probabilistically alter each suitability index variable (SIV) in that solution. If a given SIV in a given solution $\mathrm{Si}$ is selected for the modification, then its emigration rates $\mu$ of the other solutions is used to probabilistically decide which of the solutions should migrate.And it is randomly selected SIV to solution Si. The above occurrence is known as migration in BBO.

\section{Mutation}

In nature a habitat's HSI can varyabruptly due to arbitrary events. This phenomenon is termed as SIV mutation, and probabilities of species count are used to decide mutation rates. This possibility mutates low HSI as well as high HSI solutions. Mutation of high HSI solutions gives them the possibility to further improvement. Mutation rate is obtained using following equation.

$$
M(s)=m_{\max }\left(1-\frac{P_{s}}{P_{\max }}\right)
$$

Where, mmax is a user-defined parameter called mutation coefficient.

\section{Artificial Bee Colony $(A B C)$ technique}

Artificial Bee Colony (ABC) Algorithm is an optimization algorithm based on the intellectual foraging behavior of honey bee swarm. The colony of artificial bees consists of three groups of bees: employed bees, onlookers and scouts. An employed bee searches the target where the food is available. They accumulate the food and returns back to its source where they carry out waggle dance depending on the amount of food available at the target. The onlooker bee observe the dance and follows employed bee depends on the probability of the available food means more onlooker bee will follow the employed bee associated with the target having more amount of food. The employed bee whose food source becomes abandoned then it changes into a scout bee and it will search for the new food source. For solving optimization problems the population is alienated into two parts consisting of employed bees and onlooker bees. An employed bee searches the solution in the explore space and the value of objective function associated with the solution is the amount of food linked with that solution. Employed bee updates its position using Equation (27) and it updates new position if it is better than the preceding position.

$$
v_{i j}=x_{i j}+R_{i j}\left(x_{i j}-x_{k j}\right)
$$

Where vij is the new position of employee bee, $x i j$ is the current position of employed bee, $\mathrm{k}$ is a random number between $(1, \mathrm{~N}$ (population size)/2) $\neq \mathrm{i}$ and $\mathrm{j}=1,2, \ldots$, Number of design variables. $\mathrm{Rij}$ is a random number between $(-1,1)$.

An onlooker bees prefer a food source depending on the probability value related with that food source, pi , calculated using Equation (28).

IJEEI Vol. 2, No. 2, June 2014 : 86 - 95 
$P_{i}=\frac{F_{i}}{\sum_{n=1}^{N / 2} F_{n}}$

Where $\mathrm{Fi}$ is the fitness value of the solution $\mathrm{i}$ and $\mathrm{N} / 2$ is the number of food sources which is equal to the number of employed bees.

The Employed bee whose location of the food source cannot be enhanced for some predetermined number of cycles than that food source is called abandoned food source. That employed bee becomes scout and searches for the new solution randomly using Equation (29).

$$
x_{i}^{j}=x_{\text {min }}^{j}+\operatorname{rand}(0,1)\left(x_{\text {max }}^{j}-x_{\text {min }}^{j}\right)
$$

\section{HBBABC: Hybrid Biogeography Based Artificial Bee Colony Optimization}

$A B C$ is superior at exploring the search space and locating the region of global minimum. On the other hand, BBO has a high-quality exploitation searching tendency for global optimization. Based on these considerations, in order to capitalize on the exploration and the exploitation HBBABC approach is projected. Step by step methodology for the implementation of $\mathrm{HBBABC}$ for solving optimal reactive power dispatch problem is given as follows.

Step 1: Initialize BBO and ABC parameters which are essential for the algorithm to progress. These parameters include population size, number of generations essential for the termination criteria, Maximum immigration and emigration rates, number of design variables and relevant range for the design variables.

Step 2: producearbitrary population equal to the population size. Each population member has the value of all the design variables. This value of design variable is arbitrarily generated in between the design variable range specified. Each design variable in the population indicates SIVs for that particular population member (Habitat).

Step 3: acquire the value of objective function for all population members. The value of objective function so obtained designates the HSI for that Habitat (population member).

Step 4: plot the value of HSI to obtain the species count. For reactive power dispatch optimization problem is of minimization type than low HSI member is given high species count

Step 5: alter population by using the migration operator considering its immigration and emigration rates. If a given solution is selected to be customized, then its immigration rate $\lambda$ is used to probabilistically modify each suitability index variable (SIV) in that solution. If a given SIV in a given solution $\mathrm{Si}$ is selected to be modified, then its emigration rates $\mu$ of the other solutions is used to probabilistically decide which of the solutions should migrate its randomly selected SIV to solution Si. Code for migration is given in figure 1.

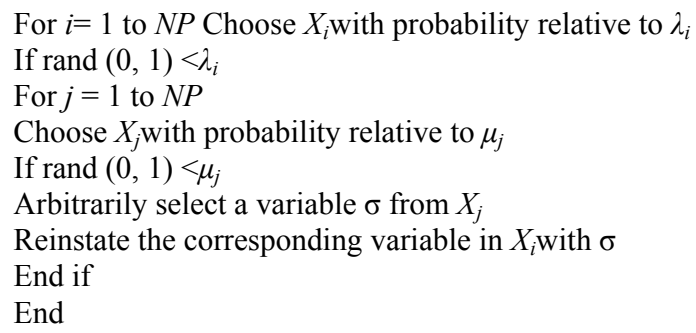

Figure 1. Code for migration

Step 6: separate the population into two equal parts to act as employed bees and onlooker bees. Attain the value of objective function for employed bees. The value of objective function so obtained indicates the amount of nectar (food) associated with that target (food source).

Step 7: renew the location of employed bees using Equation (27). If the value of objective function of the new solution is superiorto the existing solution, reinstate the existing solution with the new one. 
Step 8: compute probability related with the different solutions using Equation (28). Onlooker bee goes behind a solution depending on the probability of that solution. So more the probability of the solution more will be the onlooker bee following that solution.

Step 9: renew the position of onlooker bees using Equation (27). If the rate of objective function of the new solution is better than the existing solution, reinstate the existing solution with the new one.

Step 10: discover abandon solution and reinstate it with the newly generated solution using Equation (29). reached.

Step 11: carry on all the steps from step 3 until the specified number of generations are figure 2

Detailed HBBABC code applied to optimal reactive power dispatch problemis given in

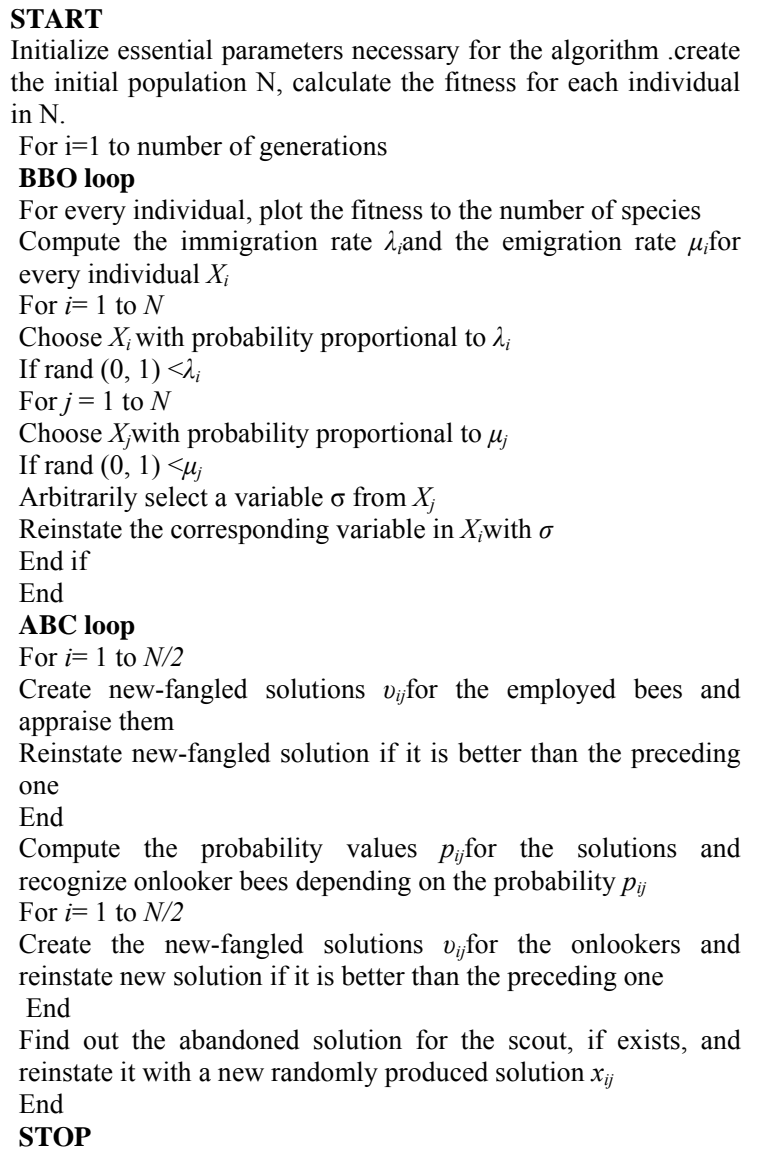

Figure 2. HBBABC code for solving optimal reactive power dispatch problem

\section{Simulation Results}

The performance of the proposed HBBABC method is demonstrated by testing it on standard IEEE-30 bus system. The IEEE-30 bus system has 6 generator buses, 24 load buses and 41 transmission lines of which four branches are (6-9), (6-10), (4-12) and (28-27) - are with the tap setting transformers. The lower voltage magnitude limits at all buses are $0.95 \mathrm{p.u}$. and the upper limits are 1.1 for all the PV buses and 1.05 p.u. for all the PQ buses and the reference bus. The simulation results have been presented in Tables 1, 2, 3\&4. And in the Table 5 shows the proposed algorithm powerfully reduces the real power losses when compared to other given algorithms. The optimal values of the control variables along with the minimum loss obtained 
are given in Table 1. Corresponding to this control variable setting, it was found that there are no limit violations in any of the state variables.

Table 1. Results of HBBABC - ORPD optimal control variables

\begin{tabular}{cc}
\hline Control variables & Variable setting \\
\hline V1 & 1.043 \\
V2 & 1.041 \\
V5 & 1.040 \\
V8 & 1.033 \\
V11 & 1.004 \\
V13 & 1.041 \\
T11 & 1.04 \\
T12 & 1.02 \\
T15 & 1.0 \\
T36 & 1.0 \\
Qc10 & 3 \\
Qc12 & 4 \\
Qc15 & 4 \\
Qc17 & 0 \\
Qc20 & 3 \\
Qc23 & 3 \\
Qc24 & 2 \\
Qc29 & 3 \\
Real power loss & 4.3258 \\
SVSM & 0.2471 \\
\hline
\end{tabular}

ORPD together with voltage stability constraint problem was handled in this case as a multi-objective optimization problem where both power loss and maximum voltage stability margin of the system were optimized simultaneously. Table 2 indicates the optimal values of these control variables. Also it is found that there are no limit violations of the state variables. It indicates the voltage stability index has increased from 0.2471 to 0.2483 , an advance in the system voltage stability. To determine the voltage security of the system, contingency analysis was conducted using the control variable setting obtained in case 1 and case 2 . The Eigen values equivalents to the four critical contingencies are given in Table 3 . From this result it is observed that the Eigen value has been improved considerably for all contingencies in the second case.

Table 2. Results of HBBABC-Voltage Stability Control Reactive Power Dispatch Optimal Control Variables

\begin{tabular}{cc}
\hline Control Variables & Variable Setting \\
\hline V1 & 1.045 \\
V2 & 1.044 \\
V5 & 1.03 \\
V8 & 1.034 \\
V11 & 1.006 \\
V13 & 1.036 \\
T11 & 0.090 \\
T12 & 0.090 \\
T15 & 0.090 \\
T36 & 0.090 \\
Qc10 & 4 \\
Qc12 & 3 \\
Qc15 & 4 \\
Qc17 & 4 \\
Qc20 & 0 \\
Qc23 & 3 \\
Qc24 & 3 \\
Qc29 & 4 \\
Real power loss & 4.9789 \\
SVSM & 0.2483 \\
\hline
\end{tabular}


Table 3. Voltage Stability under Contingency State

\begin{tabular}{cccc}
\hline SI.No & Contingency & ORPD Setting & VSCRPD Setting \\
\hline 1 & $28-27$ & 0.1410 & 0.1422 \\
2 & $4-12$ & 0.1658 & 0.1668 \\
3 & $1-3$ & 0.1774 & 0.1782 \\
4 & $2-4$ & 0.2032 & 0.2053 \\
\hline
\end{tabular}

Table 4. Limit Violation Checking Of State Variables

\begin{tabular}{ccccc}
\hline State & \multicolumn{2}{c}{ limits } & ORPD & VSCRPD \\
variables & Lower & upper & & \\
\hline Q1 & -20 & 152 & 1.3422 & -1.3269 \\
Q2 & -20 & 61 & 8.9900 & 9.8232 \\
Q5 & -15 & 49.92 & 25.920 & 26.001 \\
Q8 & -10 & 63.52 & 38.8200 & 40.802 \\
Q11 & -15 & 42 & 2.9300 & 5.002 \\
Q13 & -15 & 48 & 8.1025 & 6.033 \\
V3 & 0.95 & 1.05 & 1.0372 & 1.0392 \\
V4 & 0.95 & 1.05 & 1.0307 & 1.0328 \\
V6 & 0.95 & 1.05 & 1.0282 & 1.0298 \\
V7 & 0.95 & 1.05 & 1.0101 & 1.0152 \\
V9 & 0.95 & 1.05 & 1.0462 & 1.0412 \\
V10 & 0.95 & 1.05 & 1.0482 & 1.0498 \\
V12 & 0.95 & 1.05 & 1.0400 & 1.0466 \\
V14 & 0.95 & 1.05 & 1.0474 & 1.0443 \\
V15 & 0.95 & 1.05 & 1.0457 & 1.0413 \\
V16 & 0.95 & 1.05 & 1.0426 & 1.0405 \\
V17 & 0.95 & 1.05 & 1.0382 & 1.0396 \\
V18 & 0.95 & 1.05 & 1.0392 & 1.0400 \\
V19 & 0.95 & 1.05 & 1.0381 & 1.0394 \\
V20 & 0.95 & 1.05 & 1.0112 & 1.0194 \\
V21 & 0.95 & 1.05 & 1.0435 & 1.0243 \\
V22 & 0.95 & 1.05 & 1.0448 & 1.0396 \\
V23 & 0.95 & 1.05 & 1.0472 & 1.0372 \\
V24 & 0.95 & 1.05 & 1.0484 & 1.0372 \\
V25 & 0.95 & 1.05 & 1.0142 & 1.0192 \\
V26 & 0.95 & 1.05 & 1.0494 & 1.0422 \\
V27 & 0.95 & 1.05 & 1.0472 & 1.0452 \\
V28 & 0.95 & 1.05 & 1.0243 & 1.0283 \\
V29 & 0.95 & 1.05 & 1.0439 & 1.0419 \\
V30 & 0.95 & 1.05 & 1.0418 & 1.0397 \\
\hline & & & & \\
\hline
\end{tabular}

Table 5. Comparison of Real Power Loss

\begin{tabular}{cc}
\hline Method & Minimum loss \\
\hline Evolutionary programming[10] & 5.0159 \\
Genetic algorithm[11] & 4.665 \\
Real coded GA with Lindex as & 4.568 \\
SVSM[12] & 4.5015 \\
Real coded genetic algorithm[13] & 4.3258 \\
Proposed HBBABC method & \\
\hline
\end{tabular}

\section{Conclusion}

In this paper a novel approach HBBABCalgorithm used to solve optimal reactive power dispatch problem. The performance of the proposed algorithm demonstrated through its voltage stability assessment by modal analysis is effective at various instants following system contingencies. Real power loss has been reduced considerably and voltage profiles has been enhanced.

\section{References}

[1] O Alsac, and B Scott. "Optimal load flow with steady state security". IEEE Transaction. PAS -1973: 745-751.

[2] Lee KY, Paru YM, Oritz JL. A united approach to optimal real and reactive power dispatch. IEEE Transactions on power Apparatus and systems. 1985; PAS-104: 1147-1153 
[3] A Monticelli, MVF Pereira and S Granville. "Security constrained optimal power flow with post contingency corrective rescheduling”. IEEE Transactions on Power Systems. 1987; PWRS-2(1): 175182.

[4] Deeb N, Shahidehpur SM. Linear reactive power optimization in a large power network using the decomposition approach. IEEE Transactions on power system. 1990; 5(2) : 428-435

[5] E Hobson. 'Network consrained reactive power control using linear programming'. IEEE Transactions on power systems. 1980; PAS -99(4): 868-877.

[6] KY Lee, YM Park, and JL Oritz. "Fuel -cost optimization for both real and reactive power dispatches". IEE Proc. 131C(3): 85-93.

[7] MK Mangoli, and KY Lee. "Optimal real and reactive power control using linear programming". Electr. Power Syst. Res, 1993; 26: 1-10.

[8] SR Paranjothi and K Anburaja. "Optimal power flow using refined genetic algorithm". Electr. Power Compon. Syst. 2002; 30: 1055-1063.

[9] D Devaraj, and B Yeganarayana. "Genetic algorithm based optimal power flow for security enhancement". IEE proc-Generation.Transmission and. Distribution. 2005: 152.

[10] Wu QH, Ma JT. Power system optimal reactive power dispatch using evolutionary programming. IEEE Transactions on power systems. 1995; 10(3): 1243-1248

[11] S Durairaj, D Devaraj, PS Kannan. 'Genetic algorithm applications to optimal reactive power dispatch with voltage stability enhancement'. IE(I) Journal-EL. 2006; 87.

[12] D Devaraj. 'Improved genetic algorithm for multi - objective reactive power dispatch problem'. European Transactions on electrical power. 2007; 17: 569-581.

[13] P Aruna Jeyanthy and Dr D Devaraj. "Optimal Reactive Power Dispatch for Voltage Stability Enhancement Using Real Coded Genetic Algorithm". International Journal of Computer and Electrical Engineering. 2010; 2(4): 1793-8163.

[14] Dervis Karaboga. An Ideal Based On Honey Bee Swarm For Numerical Optimization Technical Report Tr06. 2005.

[15] Dervis Karabog, Artificial bee colony algorithm, Scholarpedia, 5(3):6915, doi:10.4249/scholarpedia.6915,2010.

[16] Dervis Karaboga, Bahriye. A comparative study of Artificial Bee Colony algorithim. Applied Mathmatics and Compution. 2009; 214: 108-132.

[17] Dervis Karaboga,Bahriye Akay,A survey algorithms simulating bee swarm intelligence, Artif Intell Rev 31:61-85 DOI 10.1007/s10462-009-9127-4,2009.

[18] Devis Karaboga, B Akay. Artifical Bee Colony (ABC). Harmony Search and Bees Algorithm on NumericalOptimization.

[19] Nadezda Stanarevic, Milan Tuba and Nebojsa Bacanin. Modified artificial bee colony algorithm for constrained problems optimization. International Journal of Mathematical Models and Methods In Applied Sciences. 2011; 3(5): 644-651.

[20] Taher N. A new fuzzy adaptive hybrid particle swarm optimization algorithm for non-linear, nonsmooth and non-convex economic dispatch problem. Applied Energy. 2010; 87: 327-339.

[21] Taher N, Babak A. An efficient hybrid approach based on PSO, ACO and k-means for cluster analysis. Applied Soft Computing. 2010; 10: 183-197

[22] Tamer A, Ali HK, Huseyin C, Gurhan G. Hybridizing the harmony search algorithm with a spreadsheet 'Solver' for solving continuous engineering optimization problems. Engineering Optimization. 2009; 41(12): $1119-1144$

[23] Thong WHT, Liu GR. An optimization procedure for truss structures with discrete design variables and dynamic constraints. Computers and Structures. 2001; 79: 155-162

[24] Victoire TAA, Jeyakumar AE. Hybrid PSO-SQP for economic dispatch with valve-point effect. Electric Power Systems Research. 2004; 71: 51-59

[25] Tatjana Davidovic', Du`san Ramljak, Milica`Selmic', Du`san Teodorovic'. Bee colony optimization for thep-center problem. Computers \& Operations Research. 2011; 38: 1367-1376.

[26] Ioná Maghali Santos de Oliveira, Roberto Schirru. Swarm intelligence of artificial bees applied to InCore Fuel Management Optimizati. Annals of Nuclear Energy. 2011; 38: 1039-1045.

[27] Simon. "Biogeography-Based Optimization". IEEE transactions on Evolutionary computation. 2008; 12(6): $702-713$

[28] Aniruddha Bhattacharya and Pranab Kumar Chattopadhyay. "Biogeography - Based Optimization for Different Economic Load Dispatch Problems". IEEE transactions on power systems. 2010; 25(2).

[29] N Sinha, R Chakrabarti, and PK Chattopadhyay. "Evolutionary programming techniques for economic load dispatch". IEEE transactions On Evolutionary Computation. 2003; 7(1): 83-94.

[30] M Vanitha, K Thanushkodi. "An Efficient technique for solving the Economic Dispatch Problem using Biogeography Algorithm". ISSN 1450-21X. 2011; 50(2): 165-172 